\title{
Tracking down the path of memory: eye scanpaths facilitate retrieval of visuospatial information
}

\author{
Agata Bochynska $^{1}$ Bruno Laeng ${ }^{2}$
}

Published online: 11 August 2015

(C) The Author(s) 2015. This article is published with open access at Springerlink.com

\begin{abstract}
Recent research points to a crucial role of eye fixations on the same spatial locations where an item appeared when learned, for the successful retrieval of stored information (e.g., Laeng et al. in Cognition 131:263-283, 2014. doi:10.1016/j.cognition.2014.01.003). However, evidence about whether the specific temporal sequence (i.e., scanpath) of these eye fixations is also relevant for the accuracy of memory remains unclear. In the current study, eye fixations were recorded while looking at a checkerboard-like pattern. In a recognition session $(48 \mathrm{~h}$ later), animations were shown where each square that formed the pattern was presented one by one, either according to the same, idiosyncratic, temporal sequence in which they were originally viewed by each participant or in a shuffled sequence although the squares were, in both conditions, always in their correct positions. Afterward, participants judged whether they had seen the same pattern before or not. Showing the elements serially according to the original scanpath's sequence yielded a significantly better recognition performance than the shuffled condition. In a forced fixation condition, where the gaze was maintained on the center of the screen, the advantage of memory accuracy for same versus shuffled scanpaths disappeared. Concluding, gaze scanpaths (i.e., the order of fixations and not simply their positions) are functional to visual memory and physical reenacting of the original, embodied, perception can facilitate retrieval.
\end{abstract}

Agata Bochynska

agata.bochynska@gmail.com

1 Department of Language and Literature, Norwegian University of Science and Technology, Trondheim, Norway

2 Department of Psychology, University of Oslo, Oslo, Norway
Keywords Visuospatial memory - Eye movements . Scanpath · Eye fixations · Eye-tracking

\section{Introduction}

Recently, a growing number of studies have pointed to the possibility of a functional role of eye movements in visuospatial memory. Research shows that eyes tend to re-fixate to the same locations as during encoding (e.g., Valuch et al. 2013; Spivey and Geng 2001; Laeng et al. 2014) and that spatial locations of eye fixations could be used as cues in memory retrieval (e.g., Hebb 1968; Hochberg 1968; Neisser 1967; Winograd and Church 1988). Additionally, in several studies a general advantage for memory performance at recognition was observed when the gaze revisited the same locations as during encoding (e.g., Foulsham and Kingstone 2012; Foulsham and Underwood 2008; Hollingworth and Henderson 2002; Holm and Mäntylä 2007; Johansson and Johansson 2014; Laeng et al. 2014; Mäntylä and Holm 2006; Stark and Ellis 1981; Underwood et al. 2009; Valuch et al. 2013). Moreover, some researchers noticed that perturbating spontaneous eye movements could disrupt memory of details about the object (Johansson and Johansson 2014; Laeng et al. 2014; Mäntylä and Holm 2006).

Importantly, this gaze behavior is not triggered by salient features in the picture in a bottom-up fashion, as shown in the studies that investigated visual imagery (e.g., Johansson et al. 2012; Laeng et al. 2014; Laeng and Teodorescu 2002). Inspired by the Brand and Stark's (1997) observations and their idea behind the Scanpath Theory, Laeng and Teodorescu (2002) conducted a study where they observed that reenactment of the similar scanpath can provide a cue for memory about a particular 
object or scene. Thus, they proposed that eye movements are functional, which is consistent with the idea that the perception is active and cognition is embodied. In the current study, we tested directly whether the serial order of eye fixations, recorded first when learning a set of checkerboard-like patterns, played a beneficial role in a recognition session ( $48 \mathrm{~h}$ later), when these were reenacted in the same, idiosyncratic, temporal sequence in which they were originally viewed. We hypothesized that a shuffled sequence of fixations, though still in their correct positions, would lead to a decrease in memory accuracy or in its efficiency (i.e., a lengthening of response times).

\section{Methods}

\section{Participants}

Twenty-eight right-handed participants (17 females) with normal or corrected to normal vision were recruited to participate in a study of visual memory (mean age 26.35 years, $\mathrm{SD}=6.45$ ).

\section{Procedure}

Eye monitoring was obtained with an iView Remote EyeTracking Device (R.E.D.) from Senso-Motoric Instruments (SMI, Berlin, Germany) and iView 3.0 ${ }^{\circledR}$ Experiment Center software was used for data collection and stimulus presentation. Every testing session was preceded with a standard calibration procedure. Testing took place in the Cognitive Laboratories at the Institute of Psychology, University of Oslo. Every participant took part in two testing sessions (encoding and recognition) with $48 \mathrm{~h}$ delay. In the first session participants were looking at 32 images of $5 \times 5$ grids resembling checkerboards where 4 black squares formed a random pattern and the rest remained white. Figure 1 represents the details of procedure in the encoding session. Participants were instructed to memorize the patterns as accurately as possible. Every time the picture disappeared from the screen they were asked to imagine the pattern once again and press the space-bar key whenever they were ready to see the next image.

Registered eye sequences from the first session determined the stimuli presentation for the second session, which was individually adjusted for every participant. Instead of static images, participants were recognizing the patterns while looking at animations where black squares appeared one after another in their correct positions but in two types of sequences-either according to the original sequence of participant's eye movements from the first session or in a shuffled sequence (Fig. 2). Additionally, patterns from the encoding session were mixed with 32

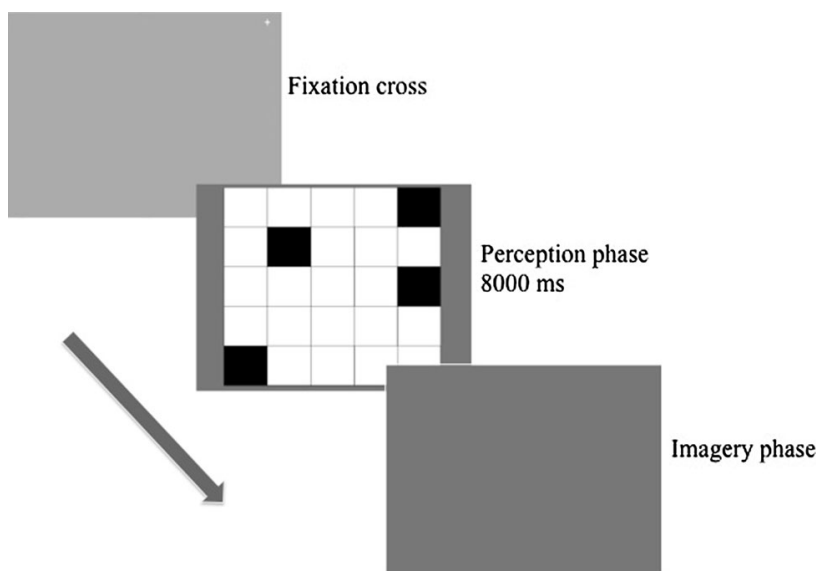

Fig. 1 Illustration of the encoding session procedure with perception and imagery phase

additional (novel) patterns presented in random sequences. When the picture disappeared, participants pressed ' $M$ ' key on the keyboard for 'old' patterns or ' $\mathrm{Z}$ ' key for 'novel' patterns. To additionally manipulate the involvement of the eye movements, recognition session was divided into two separate blocks-free viewing (participants were allowed to freely follow the appearing squares) and forced fixation (participants were asked to fixate their gaze in the middle of the screen).

\section{Results}

\section{Behavioral results}

The accuracy and RT data were computed by means of SMI $\mathrm{BeGaze}^{\circledR}$ analysis software for each participant. A repeatedmeasures $2 \times 2$ ANOVA on the mean percentages of accuracy was conducted with Condition (forced fixation and free viewing) and Sequence (same and shuffled) as withinsubjects factors. This analysis revealed a main effect of Sequence in the task, $F(1,27)=5.207, p=.03$ (see Fig. 3). Additionally, accuracy was significantly above chance when patterns were viewed according to the 'same' sequence in the free viewing condition, $t(1,27)=2.751, p=.01$, and not when the sequence was 'shuffled' (in the same condition), $t(1,27)=.102, p=.92$.

Similarly as for the accuracy data, a repeated-measures $2 \times 2$ ANOVA for the mean RTs was performed with Condition (forced fixation and free viewing) and the Sequence (same and shuffled) as within-subjects factors. The analysis revealed an interaction effect of Condition $*$ Sequence, $F(1,27)=4.224, p=.04$ (see Fig. 3). Paired samples $t$ test on mean response times revealed significant difference between 'same' and 'shuffled' sequence in the free viewing condition (with longer 
Fig. 2 Illustration of the recognition session procedure with baseline, serial presentation of the pattern and response display

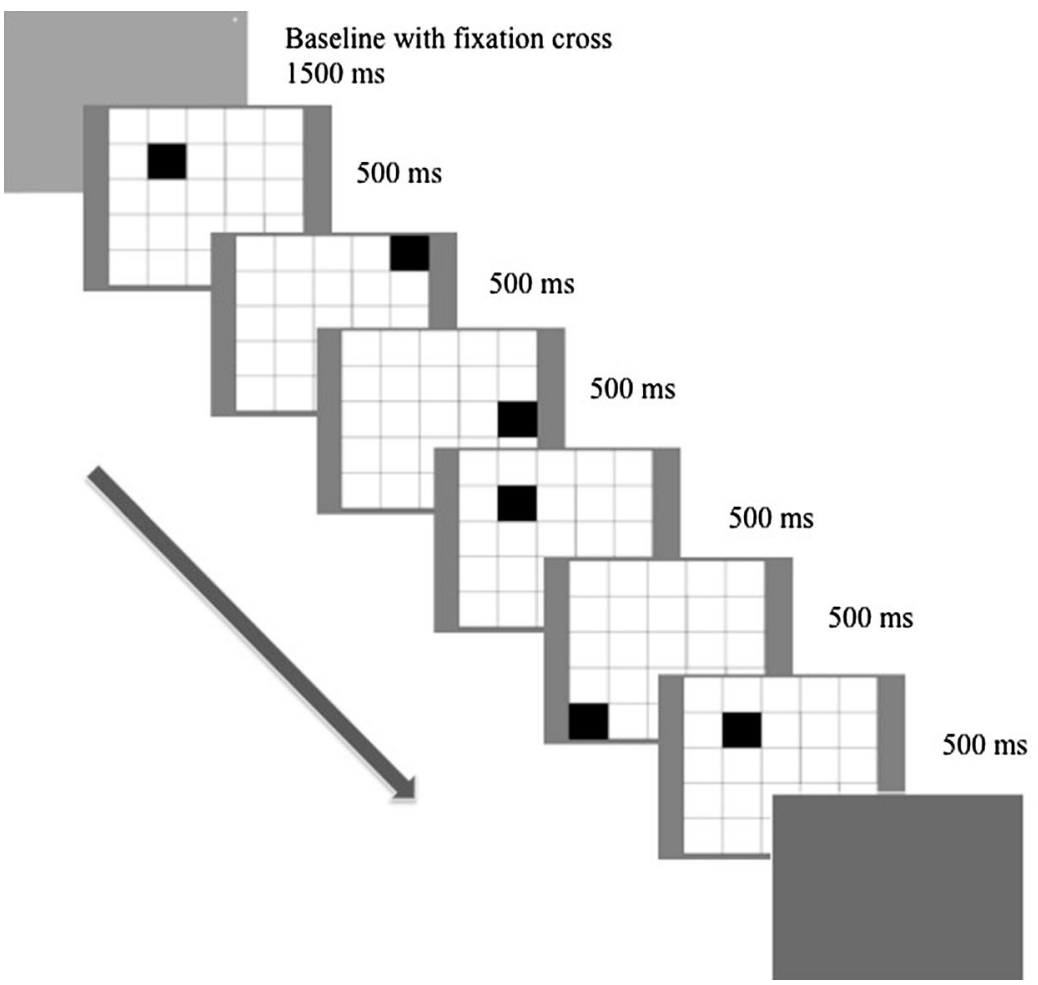

Response

response times in the latter comparing to the former sequence), $t(1,27)=-2.356, p=.026$, but not in the forced fixation condition, $t(1,27)=.131, p=.896$.

\section{Pupillary results}

We also obtained pupillary measurements as an estimate of mental effort (Kahneman 1973; Kahneman and Beatty 1966; see also Alnæs et al. 2014) in the two conditions, since some authors have argued that memory could be better in free viewing than forced fixation, because the latter condition may be more taxing for attentional capacity (cf. Martarelli and Mast 2013).

Because each square covered around $6^{\circ}$ of visual angle and the amplitude of pupillary light reflexes within the region of $15^{\circ}$ of visual angle is greatest at the center region which is of about $6^{\circ}$ (Mizukawa 2009), we excluded those trials in which (159 out of 754 trials) a black square appeared at central fixation and computed mean pupillary changes in the forced fixation (mean pupillary change $=-.213 ; \mathrm{SD}=.43$ ) and free viewing (mean pupillary change $=-.287 ; \mathrm{SD}=.43$ ) and conducted a repeated-measures $2 \times 3$ ANOVA with Condition (forced fixation and free viewing) and Sequence (same, shuffled or novel) as within-subject factors on pupillary changes in the rest of the trials $(N=595)$. This analysis did not reveal any significant effects of Condition, $F(1,23)=0.898$, $p=.353$ or Sequence, $F(2,22)=2.868, p=.079$ on pupillary changes. There was no significant interaction of Condition * Sequence, $\quad F(2, \quad 22)=2.276, \quad p=.126$. Therefore, we found no evidence that forcing fixations taxed either working memory or attention more than moving the eyes freely.

\section{Discussion and conclusions}

In the current study, visuospatial long-term memory for checkerboard-like patterns was facilitated through the enactment of the similar sequences of eye movements as indicated by recognition accuracy and faster response times in the 'same' sequence only in the free viewing but not in the forced fixation condition. This supports the hypothesis about a functional role of eye movements in visual memory (Laeng and Teodorescu 2002) and is consistent with the studies that pointed to the important role of re-fixations into the original locations from encoding (e.g., Foulsham and Kingstone 2012; Holm and Mäntylä 2007; Johansson et al. 2012; Johansson and Johansson 2014; Laeng et al. 2014; Mäntylä and Holm 2006; Valuch et al. 2013). These findings also support Hebb's (1968) account that was at the foundations of the scanpath theory (Noton and Stark 1971a, b). Importantly, the current study investigated the spacetime aspect of the eye movements' involvement in visual memory, which has been disregarded in previous studies (e.g., Brandt and Stark 1997; Laeng and Teodorescu 2002). 

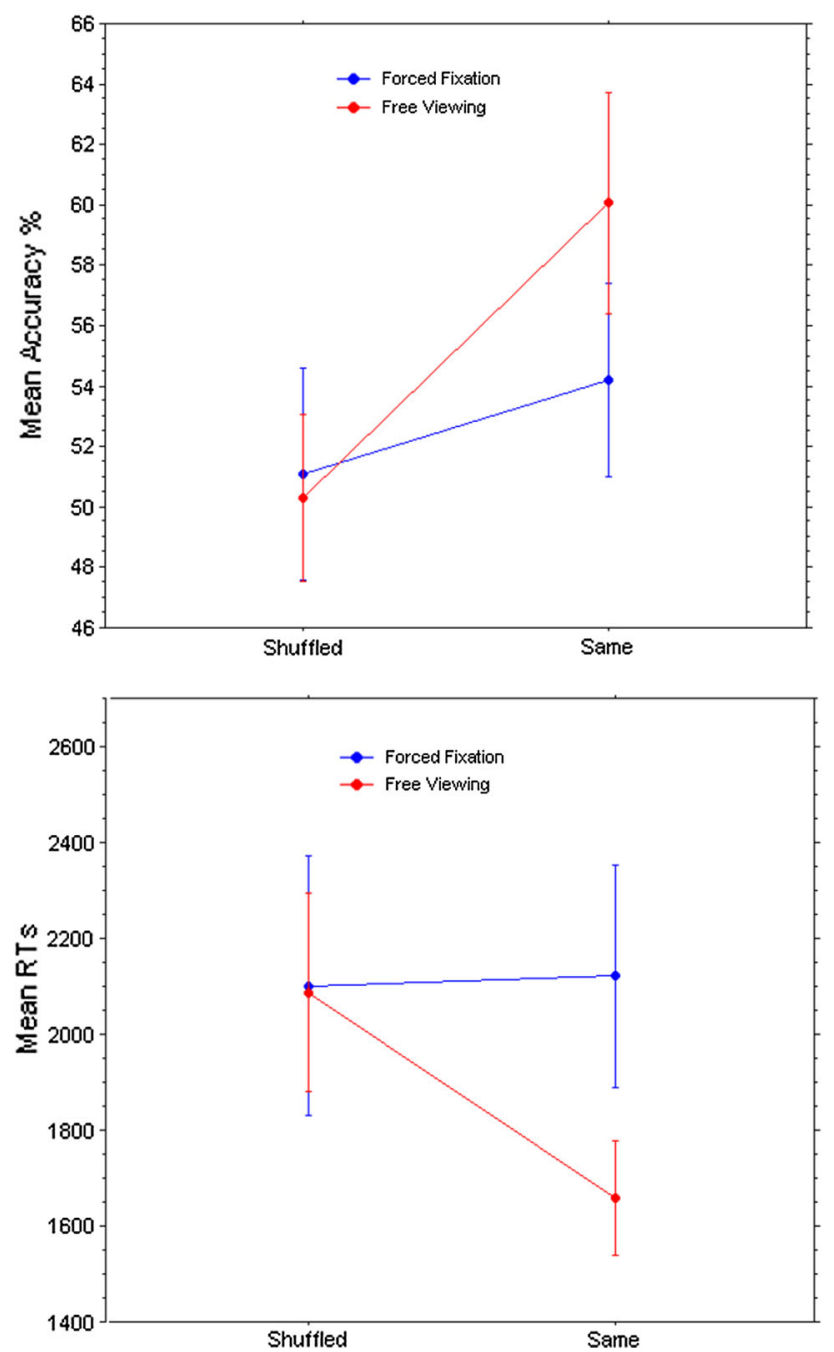

Fig. 3 Mean percentage accuracy (top panel) and mean RTs (bottom panel) in 'shuffled' and 'same' sequences shown separately for the forced fixation and free viewing conditions. The bars represent the $95 \%$ confidence intervals according to Loftus and Masson's formula (1994)

In conclusion, not just re-fixations to the original locations but also the temporal sequences of the eye movements do play a crucial role in long-term visuospatial memory.

Open Access This article is distributed under the terms of the Creative Commons Attribution 4.0 International License (http://crea tivecommons.org/licenses/by/4.0/), which permits unrestricted use, distribution, and reproduction in any medium, provided you give appropriate credit to the original author(s) and the source, provide a link to the Creative Commons license, and indicate if changes were made.

\section{References}

Alnæs D, Sneve MH, Espeseth T, Endestad T, van de Pavert SHP, Laeng B (2014) Pupil size signals mental effort deployed during multiple object tracking and predicts brain activity in the dorsal attention network and the locus coeruleus. J Vis 14(4):1, 1-20. http://www.journalofvision.org/contents/14/4/1, doi:10.1167/14. 4.1

Brandt SA, Stark LW (1997) Spontaneous eye movements during visual imagery reflect the content of the visual scene. J Cogn Neurosci 9:27-38. doi:10.1162/jocn.1997.9.1.27

Foulsham T, Kingstone A (2012) Fixation-dependent memory for natural scenes: an experimental test of Scanpath theory. J Exp Psychol Gen. doi:10.1037/a0028227

Foulsham T, Underwood G (2008) What can saliency models predict about eye movements? Spatial and sequential aspects of fixations during encoding and recognition. J Vis, 8(2):6, 1-17

Hebb DO (1968) Concerning imagery. Psychol Rev 75:466-477. doi: $10.1037 / \mathrm{h} 0026771$

Hochberg J (1968) In the minds eye. In: Hhaber RN (ed) Contemporary theory and research in visual perception. HoltRinehart \& Winston, New York, pp 309-331

Hollingworth A, Henderson JM (2002) Accurate visual memory for previously attended objects in natural scenes. J Exp Psychol Hum Percept Perform 28:113-136

Holm L, Mäntylä T (2007) Memory for scenes: refixations reflect retrieval. Mem Cognit 35(7):1664-1674. doi:10.3758/BF031 93500

Johansson R, Johansson M (2014) Look here, eye movements play a functional role in memory retrieval. Psychol Sci 25(1):236-242. doi:10.1177/0956797613498260

Johansson R, Holsanova J, Dewhurst R, Holmqvist K (2012) Eye movements during scene recollection have a functional role, but they are not reinstatements of those produced during encoding. J Exp Psychol Hum Percept Perform 38:1289-1314. doi:10. 1037/a0026585

Kahneman D (1973) Attention and effort. Prentice Hall, Englewood Cliffs

Kahneman D, Beatty J (1966) Pupil diameter and load on memory. Science 154(3756):1583-1585

Laeng B, Teodorescu DS (2002) Eye scanpaths during visual imagery reenact those of perception of the same visual scene. Cogn Sci Multidiscip J 26:207-231. doi:10.1207/s15516709 $\operatorname{cog} 2602 \_3$

Laeng B, Bloem IM, D’Ascenzo S, Tomassi L (2014) Scrutinizing visual images: the role of gaze in mental imagery and memory. Cognition 131:263-283. doi:10.1016/j.cognition.2014.01.003

Loftus GR, Masson MEJ (1994) Using confidence intervals in withinsubject designs. Psychon Bull Rev 1:476-490

Mäntylä T, Holm L (2006) Gaze control and recollective experience in face recognition. Vis Cognit 14:365-386. doi:10.1080/ 13506280500347992

Martarelli CS, Mast FW (2013) Eye movements during long-term pictorial recall. Psychol Res 77:303-309. doi:10.1007/s00426012-0439-7

Mizukawa K (2009) A trial of objective visual field measurement by pupillary reaction. Kawasaki Med J 35:51-60

Neisser U (1967) Cognitive psychology. Appleton-Century-Crofts, New York

Noton D, Stark LW (1971a) Scanpaths in eye movements during perception. Science 171:308-311. doi:10.1126/science.171. 3968.308

Noton D, Stark LW (1971b) Scanpaths in saccadic eye movements while viewing and recognizing patterns. Vis Res 11:929-942. doi:10.1016/0042-6989(71)90213-6

Spivey M, Geng J (2001) Oculomotor mechanisms activated by imagery and memory: eye movements to absent objects. Psychol Res 65:235-241. doi:10.1007/s004260100059

Stark L, Ellis SR (1981) Scanpaths revisited: cognitive models direct active looking. In: Fisher DF, Monty RA, Senders JW (eds) Eye 
movements: cognition and visual perception. Lawrence Erlbaum, Hillsdale, pp 193-227

Underwood G, Foulsham T, Humphrey K (2009) Saliency and scan patterns in the inspection of real-world scenes: eye movements during encoding and recognition. Vis Cognit 17(6/7):812-834

Valuch C, Becker SI, Ansorge U (2013) Priming of fixations during recognition of natural scenes. J Vis 13(3):1-22. doi:10.1167/13. 3.3
Winograd E, Church V (1988) Role of spatial location in learning face-name associations. Mem Cognit 16(1):1-7. doi:10.3758/ BF03197739 